\title{
Frontières
}

\section{Des rites funéraires}

\section{Pour mieux vivre et faire vivre la dernière traversée}

\section{Nicole Bouchard et Mario Bélanger}

Volume 16, numéro 2, printemps 2004

Deuil, blessure vive

URI : https://id.erudit.org/iderudit/1074117ar

DOI : https://doi.org/10.7202/1074117ar

Aller au sommaire du numéro

\section{Éditeur(s)}

Université du Québec à Montréal

ISSN

1180-3479 (imprimé)

1916-0976 (numérique)

Découvrir la revue

Citer cet article

Bouchard, N. \& Bélanger, M. (2004). Des rites funéraires : pour mieux vivre et faire vivre la dernière traversée. Frontières, 16(2), 53-57.

https://doi.org/10.7202/1074117ar
Résumé de l'article

Cet article présente les grandes lignes d'une recherche-action menée auprès d'une entreprise funéraire de la région du Saguenay-Lac-Saint-Jean. Après avoir décrit le contexte de la recherche et son déroulement, les auteurs relatent les possibles ouverts par cette recherche en présentant une série de dix interventions rituelles proposées à des familles vivant un deuil difficile. Ces premiers résultats de recherche pourront contribuer à mieux documenter le débat autour de l'urgence du travail de recomposition des rites funéraires.
Ce document est protégé par la loi sur le droit d'auteur. L'utilisation des services d'Érudit (y compris la reproduction) est assujettie à sa politique d'utilisation que vous pouvez consulter en ligne.

https://apropos.erudit.org/fr/usagers/politique-dutilisation/ 


\section{Résumé}

Cet article présente les grandes lignes d'une recherche-action menée auprès d'une entreprise funéraire de la région du Saguenay-Lac-Saint-Jean. Après avoir décrit le contexte de la recherche et son déroulement, les auteurs relatent les possibles ouverts par cette recherche en présentant une série de dix interventions rituelles proposées à des familles vivant un deuil difficile. Ces premiers résultats de recherche pourront contribuer à mieux documenter le débat autour de l'urgence du travail de recomposition des rites funéraires.

Mots clés : rite - deuil - mort -

Saguenay-Lac-Saint-Jean.

\section{Abstract}

This article presents highlights from a terrain-study of a funeral home in the region of Saguenay-Lac St. Jean. After describing the research context and method, the authors present the possibilities opened up by this research by presenting a series of ten ritual interventions proposed to the families living a difficult grieving process. These initial research results should contribute to a better documentation of the debate over the urgency to work on the recomposition of funeral rites.

Key words: ritual - mourning - death Saguenay-Lac St. Jean.

\section{DES RITES FUNÉRAIRES Pour mieux vivre et faire vivre la dernière traversée}

\section{Nicole Bouchard, Ph.D., professeure au Département des sciences humaines, Université du Québec à Chicoutimi. \\ Mario Bélanger, Ph.D., professeur au Département des sciences humaines, Université du Québec à Chicoutimi.}

Dans de nombreuses sociétés occidentales, les religions instituées, tout comme nombre d'autres lieux d'expression et de façonnement de l'identité, ne semblent plus réussir à fournir d'espaces de construction $\mathrm{du}$ sens. Nous entendons par ce terme des lieux où s'élaborent collectivement des modèles d'interprétation. Ceux-ci permettent, entre autres, d'expliquer les aléas de l'existence et de les assumer. Les rites funéraires, en tant qu'espaces collectifs d'interprétation de la mort, ne semblent pas avoir échappé à cette crise du sens.

La gestion du mourir en l'espace de quelques décennies s'est considérablement déplacée. La traditionnelle route des morts que des générations de Québécois et de Québécoises ont empruntée est aujourd'hui devenue une autoroute à voies rapides et aux échangeurs multiples. Le continuum rituel, qui allait de la prise en charge de la personne mourante (toilette funéraire, onction des malades, exposition à la maison) jusqu'à l'inhumation et au service anniversaire, s'est fragmenté (Bouchard, 1992; Doyon, 1954). Tout se passe comme si les modèles d'interprétation et les symboles, qui en constituent la trame implicite, étaient devenus opaques aux yeux de ceux qui y trouvaient encore du sens il n'y a pas si longtemps. Deux phénomènes, fort éloquents à ce chapitre, sont à considérer ici. Le premier est l'éclatement interne des rites qui se traduit par une brisure de la mémoire où chacun est souvent laissé à lui-même dans le travail de construction de sa propre interprétation. Le deuxième est l'éclatement externe des rites qui se traduit par une tendance lourde à la diminution constante des temps disponibles et de l'espace d'expression des rituels (Lemieux, 1995, p. 32-33). Cette individualisation des pratiques rituelles et leur disparition progressive de la scène sociale et ecclésiale comportent des enjeux et des incidences sur le vécu du deuil. Comment dire l'absence de la séparation? Comment traduire la douleur de perdre un être cher? Comment aider ceux qui se préparent à nous quitter à mieux préparer la traversée ? Enfin, comment guérir et vivre avec le silence de ceux que l'on a aimés? Il nous semble que la question des rituels funéraires constitue un enjeu majeur qui traverse l'ensemble de notre culture. Qu'il nous suffise d'évoquer, à ce titre, le débat entourant les funérailles du peintre Riopelle et, plus récemment, de Pierre Bourgeault. Cette problématique, il va sans dire, déborde largement la question de l'attachement ou non à l'une ou l'autre religion. La question des rites funéraires constitue un enjeu non seulement théologique ou thérapeutique mais aussi anthropologique. C'est de l'humain qu'il est 
question et de sa capacité à supporter et à traverser l'épreuve de la maladie, de la mort et de la souffrance. Ainsi, la thématique du deuil concerne plusieurs milieux de pratique qui doivent intervenir et proposer des parcours pour soulager et faciliter les nombreux et multiples passages qu'oblige la confrontation à la mort dans une culture qui s'obstine à la nier.

Dans le cadre de cet article, nous présenterons l'apport de la réflexion anthropologique sur les rites de passage pour l'intervention auprès de personnes endeuillées. Depuis plusieurs décennies, les approches psychologiques ont occupé l'avant-scène des pratiques. Tout en reconnaissant l'apport indéniable de cette discipline, nous proposons de réfléchir sur l'apport original et complémentaire de l'anthropologie. Nous présenterons les grands moments d'une recherche-action menée en partenariat avec une importante entreprise funéraire de la région du Saguenay-Lac-Saint-Jean. Ce partenariat original et novateur nous a permis de partager le quotidien de ce milieu, d'écouter et d'échanger avec le personnel et d'intervenir auprès des familles touchées par la mort. Nous insisterons principalement sur la description de la recherche et son cadre d'intervention. Nous sommes conscients qu'un tel choix rédactionnel comporte certaines limites dans la mesure où il laisse peu d'espace pour le développement du travail de théorisation qui accompagne ce devis de recherche.

\section{CONTEXTE ET PROBLÉMATIQUE DE LA RECHERCHE}

Au départ de ce parcours de recherche, on ne retrouvera pas la lecture d'un ou des ouvrages sur les rites ou encore un intérêt de recherche sur les rituels funéraires. En fait, tout a commencé par un coup de fil émanant d'un responsable d'une entreprise funéraire, en juin 1999 :

Nous avons actuellement à prendre en charge une famille dont le bébé de neuf mois vient de décéder accidentellement. Les parents, quoique baptisés, se disent athés et ne veulent pas faire appel au service d'un prêtre pour obtenir du soutien. Que pouvons-nous faire pour les aider?

Et nous avons été touchés par cette demande. Surpris par le sens du professionnalisme des dirigeants de cette entreprise. Ensemble, nous avons accompagné ce jeune couple. Des gestes ont été posés, des paroles ont été échangées. Les parents, pendant quelques jours, sont venus au salon funéraire bercer leur enfant. Pour l'occasion, nous avions aménagé une petite pièce où se trouvait une chaise berçante, une chaîne stéréo, une couverture. Ces moments d'intimité entre les parents et leur enfant décédé ont été difficiles à supporter et à vivre pour le personnel de l'entreprise, peu habitué à s'impliquer autant dans le travail de soutien et d'accompagnement. Au fil des mois, cette demande de collaboration et de soutien a été suivie par plusieurs autres. Des liens se sont tissés, la confiance s'est installée et l'idée d'un partenariat de recherche sur la question des rites funéraires et de l'urgence de leur recomposition au sein de ce milieu a vu le jour.

Les premiers mois de nos échanges ont été occupés à la clarification du rôle des intervenants du milieu funéraire dans le champ de la ritualité. Pour plusieurs, le rituel se limitait à l'espace de la célébration en présence d'un meneur de rites rattaché à une institution religieuse. On ne surprendra personne en affirmant la place prépondérante de l'Église du Québec dans le soutien et la traversée du deuil. Il n'est pas lointain ce temps où le curé assistait et accompagnait tous les grands moments de ce passage allant de l'onction des malades, qui marquait le début du travail de séparation, jusqu'à la célébration du service anniversaire, un an après le décès. Il nous semble que notre culture n'a pas encore pris suffisamment conscience du vide laissé par la dissolution et la dispersion des rites assumés par la sphère religieuse.

Qui administre l'expérience du sacré en l'absence d'institution religieuse? Les individus arriveront-ils, de leur propre ressort, à trouver des lieux d'administration de leur expérience du sacré et à lui donner un sens ? [...] Si les limites de la condition humaine ne sont plus administrées par un clergé, si un clergé ne veille plus à l'application des règles rituelles, qui s'occupera de maintenir des interdits, de les rappeler lors de rituels récitations, d'encadrer les expériences du sacré et de les reconnaître en leur assignant un sens? (Jeffrey, 2003, p. 216-217)

Intervenant en première ligne, l'entreprise funéraire constitue un observatoire privilégié des déplacements du sacré et de sa migration vers des espaces plus séculiers. Notre insertion dans ce milieu de pratique nous a permis de constater comment se vivent les «métamorphoses» du sacré qu'évoquent plusieurs auteurs contemporains (LeBreton, 2000; Ménard, 1999; Jeffrey, 1998; Bastide, 1975). En ce qui concerne le milieu funéraire, on a publié des études très critiques à l'égard de cette tentative d'institutionnalisation du sens au sein d'une entreprise privée ou à propriété collective. En effet, plusieurs recherches fondamentales démontrent que le milieu des entreprises funéraires est peu ou mal préparé à accueillir de telles demandes. Affranchie de toute orthodoxie liturgique, la structure organisationnelle de l'industrie permettrait au client de créer un rituel sur mesure, ajusté aux références et aux croyances éclatées de son répertoire identitaire, laissant ainsi l'individu seul, sans l'appui que pourrait lui procurer un consensus social. Entre les funérailles à la carte et les funérailles solitaires, nous ferions face à une marchandisation du sens de la mort révélant la fragilisation des liens sociaux et la fragmentation de notre culture (St-Onge, 2001 ; Bacqué, 1997).

Même si nous assumons une bonne part de ces critiques, notre implication dans le quotidien d'une entreprise funéraire nous a permis de nuancer la sévérité de ces diagnostics. À côté de la logique marchande qui souvent et à juste titre fait l'objet de nombreuses critiques, nous pouvons discerner une logique du don et un souci véritable d'offrir un accompagnement de qualité aux personnes touchées par la mort d'un proche. C'est sur ce versant que nous avons voulu inscrire cette démarche de recherche, démarche qu'il convient maintenant de mieux déployer.

\section{MÉTHODOLOGIE ET \\ DÉROULEMENT DE LA RECHERCHE}

L'expertise développée par notre équipe et l'objet même de la recherche, qui englobe l'idée de participation, de transformation de discours et d'action, sont intimement liés au cadre de travail de la recherche-action. Au plan épistémologique, ce choix se justifie par l'objet même de la recherche qui vise la transformation dans la pratique et les discours des personnes travaillant dans le milieu des entreprises funéraires, afin que leurs interventions soient mieux adaptées au contexte culturel et social des familles qui constituent leur clientèle.

La recherche-action vise un changement par la transformation réciproque de l'action et du discours, c'est-à-dire d'une action individuelle à un pratique collective efficace et incitatrice, et d'un discours spontané à un dialogue éclairé voire engagé. Elle exige qu'il ait un contrat ouvert, formel (plutôt non structuré) impliquant une participation coopérative pouvant aller jusqu'à la cogestion.

(Morin, 1990, p. 80)

Le premier moment de notre démarche a permis d'établir un langage commun entre les chercheurs et les collaborateurs (employés et gestionnaires de l'entreprise). Des séances de travail et d'échange avec tout le personnel (deux journées) ont été l'occasion de présenter le projet. Mais plus important encore, ces rencontres ont permis 


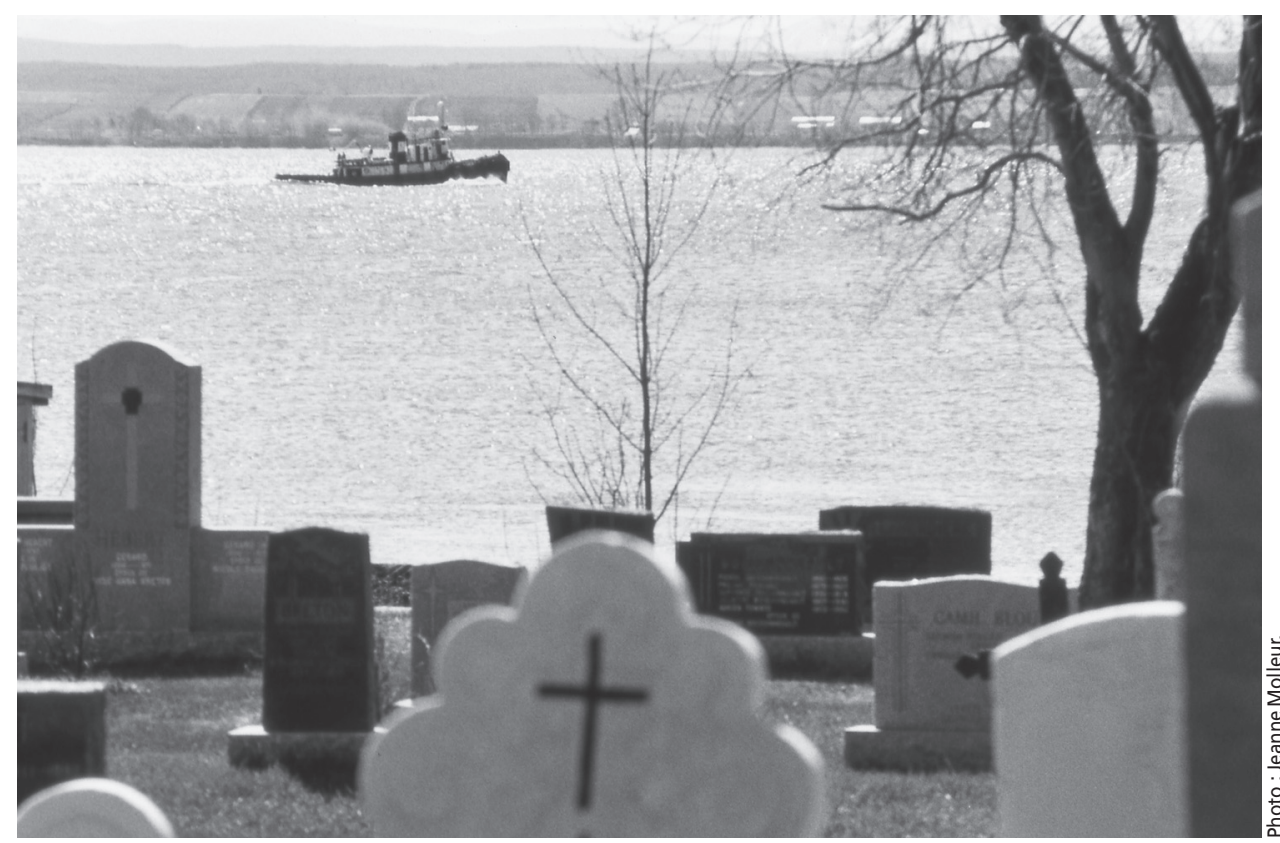

de recueillir les observations concernant les perceptions des membres du personnel de l'entreprise et les différentes significations qu'ils donnent à la pratique actuelle.

$\mathrm{Au}$ plan de la conduite de la recherche, nous avons dû réfléchir sur la place que doivent occuper les chercheurs au sein de l'entreprise. Il s'agit de participer et de s'intégrer à une pratique déjà en cours en évitant à tout prix la confusion des rôles et des fonctions. Des rencontres hebdomadaires ont été tenues et ont permis la réalisation d'un protocole d'intervention permettant de bien délimiter les rôles de chacun, de fixer les critères liés aux choix des informateurs et informatrices.

C'est ainsi qu'il a été établi que le choix des familles ${ }^{1}$ participantes serait fait lors de l'entrevue d'accueil menée par le directeur de l'entreprise. Cette personne a offert aux membres de la famille la possibilité de participer à la recherche sur une base volontaire en acceptant de recevoir un soutien en matière de ritualité. C'est à ce second moment que l'équipe de recherche ${ }^{2}$ entre en scène et procède à une entrevue exploratoire avec des membres désignés de la famille. D'une durée d'environ deux heures cette rencontre permet de recueillir les matériaux discursifs et symboliques qui formeront la matière première de l'élaboration rituelle. Il s'agir de créer un espace pour que les membres de la famille prennent le temps de faire le récit de vie de la personne décédée, de relater les étapes importantes de sa vie, d'identifier les moments plus difficiles, d'échanger sur l'héritage affectif qu'elle laisse à ses proches. Ce récit est par la suite retranscrit et analysé afin de permettre un certain décryptage de la «vision du monde» partagée par les individus au sein de leur groupe d'appartenance. L'ana- lyse à laquelle nous procédons comporte deux étapes qui sont relativement proches de celles évoquées par les sémioticiens. La première étape consiste à mettre au jour les grands thèmes qui organisent le discours des informateurs. La seconde étape consiste à relire l'histoire et à saisir l'articulation logique qui lie les grands schèmes organisateurs du récit. Notre objectif est de découvrir le sens que ces personnes donnent à l'épreuve et dans quelle mesure ce sens se prolonge dans les moyens qu'elles se donnent pour l'assumer. C'est sur ce travail d'écoute et d'analyse que s'élabore la construction de parcours rituels. Ces parcours sont assumés par les responsables de la recherche, en présence d'un intervenant de l'entreprise. Comme chercheur, nous trouvons important de nous impliquer dans l'élaboration rituelle dans la mesure où notre démarche implique un suivi auprès des familles. Nous sommes conscients des risques que comporte une telle pratique mais n'est-il pas lié au défi même de la recherche-action et de l'observation participante? Comment avons-nous procédé à ce travail ? Quelles clés de compréhension guident notre intervention?

\section{LA STRUCTURE TERNAIRE DES RITES : UNE GRILLE D'INTERVENTION PERTINENTE}

Notre intervention s'alimente aux travaux d'Arnold van Gennep et de son ouvrage célèbre publié en 1909 sur Les rites de passage. Nous ne pouvons reprendre l'ensemble de l'œuvre de cet ethnologue français ; nous nous contenterons ici de résumer très brièvement les paramètres qui ont alimenté notre pratique. Un lecteur intéressé à approfondir aura avantage à parcourir un ouvrage collectif publié en 1994 par Thierry
Goguel d'Allondas qui demeure une référence en la matière et qui s'intitule: Rites de passage: d'ailleurs, ici pour ailleurs.

Dans ses travaux, Arnold van Gennep insiste sur la notion de séquence cérémonielle. Il s'intéresse avant tout à ce qu'on pourrait appeler une logique de l'action. Il recommande ainsi d'éviter de traiter isolément tel ou tel élément du rituel. Il lui paraît nécessaire, pour en interpréter la signification, de replacer ces éléments dans un processus d'ensemble dont le déploiement produit une forme temporelle, rendue signifiante et opérante par une disposition séquentielle contraignante. L'application de la séquence cérémonielle à toutes sortes de situations constituait en fait une véritable sécularisation du concept, que l'on ne pouvait plus lier nécessairement au seul domaine de la religion. Ainsi s'ouvrait le champ d'une ritologie générale, située en amont de la différenciation religieuse, même si l'on devait reconnaître que cette dernière ne pouvait guère s'opérer sans le moyen du processus rituel.

L'apport théorique de Van Gennep pourrait se résumer en cette phrase : un passage ne se fait pas en deux temps, mais en trois. Pour en définir les trois phases, l'auteur emploie une double terminologie, l'une plus fonctionnelle qui se décompose en rites de séparation, rites de marges et rites d'agrégation et une autre plus formelle, constituée de rites préliminaires, liminaires et postliminaires. Les termes employés dans cette seconde formulation mettent en évidence d'une manière lexicalement très heureuse l'importance de la marge, de la phase intermédiaire. D'ailleurs cette notion de liminalité, de seuil, de passage, se révélera comme une des plus fécondes de l'anthropologie ultérieure, comme en témoigne l'œuvre de Victor Turner qui confirme la portée générale, voire universelle des travaux de van Gennep.

La prise en compte de ces travaux est à l'origine de l'élaboration de notre cadre d'intervention. Dans un premier temps, nos efforts ont porté sur le dévoilement d'une séquence cérémonielle déjà à l'œuvre dans la pratique. Avec les intervenants du milieu, nous avons identifié cinq moments porteurs qui marqueraient les premiers contours d'une séquence cérémonielle.

- le premier contact avec la dépouille à l'ouverture du salon;

- la fermeture en soirée du salon funéraire;

- la fermeture du cercueil, comme dernier adieu et la célébration des funérailles ou célébration d'adieu; - le moment de l'incinération pour les témoins des familles et / ou la mise en terre au cimetière ou la mise en niche au colombarium; 
- le service anniversaire ou la mise en place d'une cérémonie commémorative dans l'année ayant suivi le deuil.

C'est sur cette cartographie de la pratique que notre équipe de recherche a concentré ses premières interventions. Nous sommes conscients que le parcours séquentiel lié au processus de deuil est beaucoup plus complexe et devrait débuter bien avant l'entrée de la dépouille au salon funéraire. À ce stade-ci, nous devons nous concentrer sur la section du parcours dont la responsabilité revient aux entreprises funéraires. Il est certain que nos travaux ultérieurs viseront à compléter la séquence à l'intérieur même de l'entreprise et aussi à l'extérieur de cette dernière afin d'élargir le partenariat actuel aux différents acteurs de la pratique (hôpitaux, résidences en soins palliatifs, CLSC, entreprises funéraires, Église, etc.).

\section{LA PHASE DE SÉPARATION OU LES RITES PRÉLIMINAIRES}

Cette phase est immédiatement consécutive à un événement : celui qui commande le rite. Un individu sort de son état antérieur et cette rupture demande à être soulignée, expliquée ou facilitée. Dans la plupart des rites funéraires observés, la phase de séparation est généralement peu élaborée. Dans nos sociétés, elle se résume souvent à un rassemblement de personnes proches autour de la dépouille. Nos observations démontrent un malaise autour des rites de séparation. Tout se passe comme si personne ne s'autorisait à dire, ou mieux, à dire l'inter-dit: « il nous a quittés, il est mort, il ne reviendra plus». Bien au contraire, l'analyse des pratiques dévoile une accentuation du déni de séparation. Qu'il nous suffise d'évoquer, à titre exemplaire, les montages photos de la vie du défunt, très populaires auprès des familles, mais qui, en fait, ne dévoilent que le côté lumineux de la vie du proche. Rarement nous avons vu une séquence qui montre les traits de la maladie sur le visage de celui ou celle qui nous a quittés. Les évocations autour du cercueil sont centrées sur les grands moments de la vie du disparu et rarement sur le fait qu'il n'est plus parmi nous, qu'il a franchi l'autre rive. Plus encore, nos nouvelles habitudes entourant le traitement du cadavre créent aussi une pression sur les rites de séparation. Ainsi, la diminution ou l'absence même de temps d'exposition, l'absence de contact réel avec le cadavre, le raffinement et le potentiel de «maquillage » des techniques de thanatopraxie sont autant d'obstacles à l'entrée dans ce premier moment du rite, à savoir le nécessaire travail de séparation. Dans l'espace de cette recherche, nous avons, en lien avec le personnel, entrepris un balisage des temps possibles pour dire la séparation. Nous avons ainsi identifié trois moments incontournables qui appellent un rituel. Le premier contact avec la dépouille est un moment important et qui demande un traitement. Ainsi, lorsqu'il y a exposition, la fin de la première heure d'exposition donne lieu à une prise de parole de la part du personnel. Il ne s'agit pas de faire une simple prière ou de lire un texte quelconque. À partir du récit de la famille, nous retraçons (souvent dans le cadre d'un échange) les grands moments de la vie de la personne décédée. À la suite de ce récit, un geste sera posé, geste qui veut faire mémoire des relations tissées avec cette personne. À la fin, nous invitons les familles à prendre acte $d u$ départ définitif de l'être cher. Ainsi le premier contact avec la dépouille appelle un moment rituel. Dans les cas où il n'y a pas d'exposition ou seulement l'incinération, nous insistons pour que la famille, ne seraitce que quelques minutes, soit mise en présence de la dépouille. Un espace a d'ailleurs été aménagé pour permettre de vivre ce contact dans la dignité et le recueillement. À ce moment aussi, une séquence rituelle sera mise en place pour permettre de dire et de vivre les émotions liées à la séparation. D'autres moments font l'objet d'une attention particulière puisque forte en évocations symboliques. Nous pensons ici à la fermeture du salon en soirée ou encore à la fermeture du cercueil, comme à deux moments qui appellent des paroles, des gestes, des symboles. Ainsi, nos interventions ont permis de baliser la période d'exposition en déployant des séquences rituelles qui favorisent et soutiennent le travail de séparation.

Ces quelques orientations intéresseront aussi les personnes qui travaillent dans le vaste champ de l'accompagnement des mourants. Il est important et souhaitable que ce travail de séparation puisse être amorcé déjà quand la mort s'annonce et quand le départ devient imminent. À ce titre, nos travaux pourraient très certainement soutenir les réflexions en cours sur le type d'accompagnement à fournir dès les premiers instants de la mort, voire dès que cette dernière, lorsqu'elle est prévisible, s'annonce.

\section{LA PHASE DE MARGE OU LES RITES LIMINAIRES}

La place et l'étendue de cette phase de latence ou de marge ne manqueront pas d'alimenter nos discussions et nous voulons éviter un tel débat plus théorique. On aura compris que notre intention n'est pas de faire un découpage précis de ces phases. En effet, ces dernières ont des contours très perméables et varient en fonction des expériences. Nos observations tentent de démontrer que la sortie de la période de marge est de plus en plus longue pour les personnes endeuillées. Les possibilités entourant la disposition du corps, le rétrécissement du temps d'exposition retranchent de plus en plus le temps nécessaire à l'élaboration de rites qui soient efficaces dans la traversée du deuil.

Généralement, pour les proches de la personne décédée, la phase de marge s'amorce pendant le séjour plus ou moins prolongé du corps dans le cercueil. Toutefois, sa durée varie selon les cultures de sorte qu'il est souvent difficile d'identifier le moment précis où cette phase prend fin. Dans nos sociétés, la période de marge pourrait se terminer avec l'inhumation ou l'incinération du corps, mais rien n'est moins certain. Il faut voir l'importance que prend la commémoration généralement fixée à un an post-inhumation.

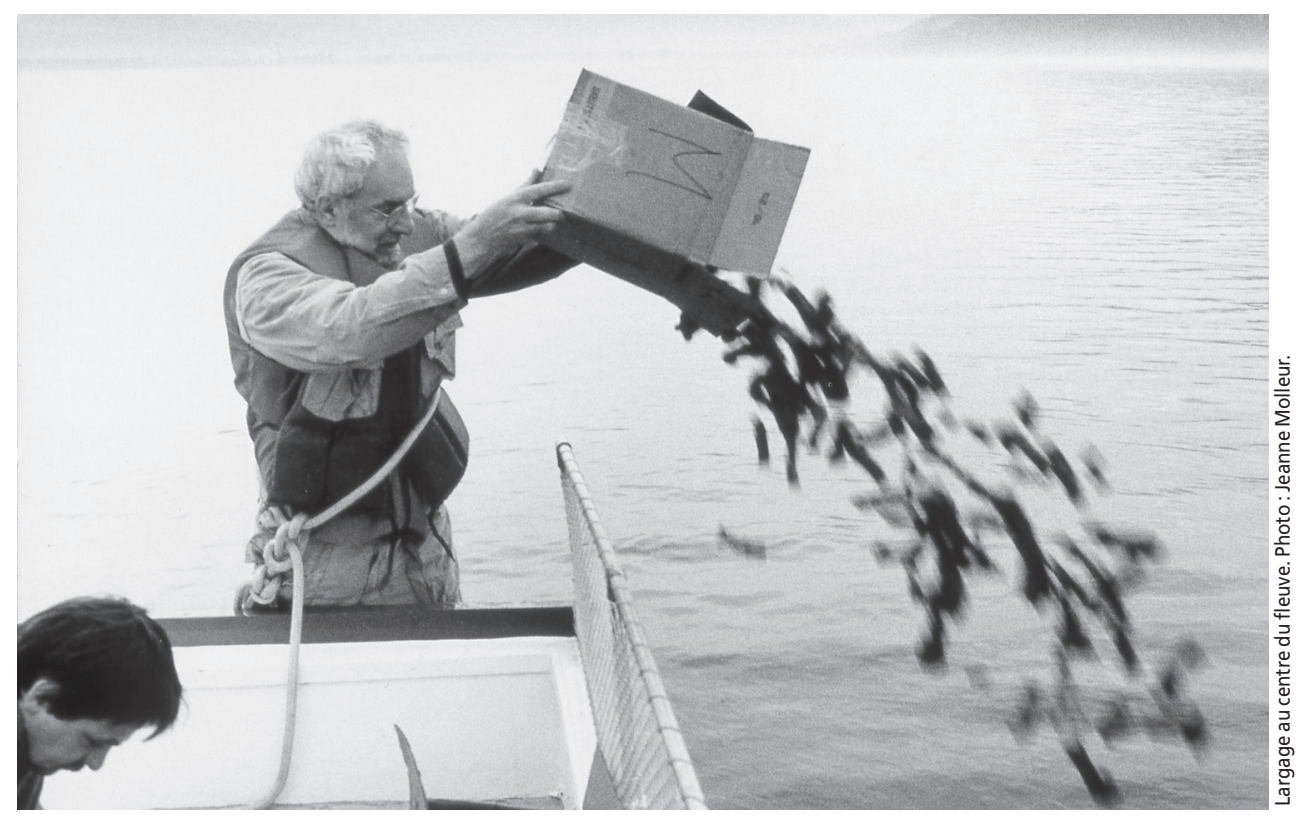


Lors de cette phase, les représentations à l'œuvre autour du départ du défunt dévoilent que ce dernier est encore très présent dans la vie des proches. Qu'il nous suffise d'évoquer les commentaires maintes fois entendus de personnes vivant un deuil : «Je sens sa [le défunt] présence»; «je lui parle»; «je sens qu'il veille sur moi»; "c'est lui qui m'envoie la force de poursuivre ». Le disparu n'appartient plus au monde des humains mais n'a pas encore atteint celui des morts. À cette phase, les personnes endeuillées vivent dans une sorte d'état intermédiaire et de latence où elles se sentent comme en dehors du temps et de l'espace. Elles vivent beaucoup d'isolement et ne sont pas encore en mesure de réintégrer leur réseau d'appartenance. Cet état liminaire est la période la plus décisive du deuil.

En ce qui concerne notre travail d'intervention, nous avons assuré un suivi auprès d'une famille qui a manifesté son intérêt à poursuivre avec nous le déroulement de la séquence rituelle. Sur une période allant de six à huit mois, nous avons mené une série de trois interventions. Le réinvestissement des symboles élaborés lors de la première phase et le développement de séquences centrées sur des symboles à fortes valences matricielles (coffre, coupe, anneaux) nous ont permis de constater l'intérêt de poursuivre dans cette voie d'intervention. Il va sans dire que nous en sommes au tout premier stade de notre réflexion et que nous nous gardons bien de tirer des conclusions trop hâtives sur une expérience aussi singulière. Nous espérons cependant que la consolidation de notre partenariat et le développement d'une deuxième phase de la recherche nous permettra de mieux déployer ce volet qui constitue en fait le cœur même de notre démarche d'intervention.

\section{LA PHASE D'AGRÉGATION OU LES RITES POSTLIMINAIRES}

Cette phase représente le moment où le défunt est réputé avoir atteint le « royaume des morts». Dans certaines sociétés, on se représente ce lieu comme un monde et une société parallèle dans lesquels le défunt prend enfin sa nouvelle place. La fin du deuil (qu'il n'est pas facile de situer dans le temps pour nos sociétés) marque généralement le retour des personnes endeuillées parmi les leurs. Il se pourrait que cela se produise au moment des cérémonies commémoratives. Il est généralement reconnu en anthropologie que ces rites servent à reconstituer le tissu social fragilisé par la mort d'un des membres de la communauté. Par ailleurs, les nouvelles habitudes en matière de disposition du corps sont venues brouiller les repères traditionnels. Mentionnons ici à titre d'exemple la pratique de certaines familles qui, à chaque Noël, reviennent chercher l'urne d'un proche décédé pour «qu'il ou elle vienne passer Noël avec nous ». Que dire aussi des possibilités technologiques qui rendent possible la confection de différents objets qui permettent de porter en permanence les cendres du disparu. Il est difficile d'évaluer les effets de ces pratiques sur la traversée du deuil. Chose certaine, il convient d'être attentif à ces nouvelles pratiques qui n'ont rien d'anodines.

Dans le cadre de notre intervention, nous avons beaucoup investi dans l'élaboration de célébrations commémoratives. Il s'agit de célébrations collectives dans lesquelles on met en scène tout le parcours et le travail des phases antérieures pour permettre la prise de conscience de la fin du deuil. Depuis deux ans, environ mille personnes ont été rejointes par ces célébrations qui ont lieu à tous les mois de novembre. Les commentaires recueillis lors de ces interventions laissent penser que ce travail correspond à un besoin important des personnes endeuillées et facilite leur retour dans le courant de la vie et de leur communauté.

Il nous semble que la description, même très brève de notre démarche, dévoile à quel point nous cherchons à nous distancier des pratiques qui offrent du «prêt-à-porter» funéraire. Sur la base de cette première expertise, l'équipe procède à l'identification d'une deuxième cohorte d'une vingtaine de familles pour le printemps et l'été 2004. Un tel développement pourra nous permettre de consolider notre partenariat et de resserrer encore nos liens avec l'entreprise funéraire participante. Un autre défi concerne l'investissement que nous devrons faire dans l'effort d'analyse et d'élaboration du cadre théorique de nos travaux. Présentement, nous étudions les impacts possibles des travaux de Gilbert Durand sur l'élaboration des séquences rituelles. Un dernier défi concerne la structuration de notre démarche. Il faut aussi créer des ponts entre les différents intervenants qui œuvrent auprès des personnes touchées par la mort d'un proche. Présentement, chacun travaille isolément et est confiné à son seul espace. Nous nous prenons quelquefois à rêver de ce que pourrait accomplir une équipe de travail qui réunirait des intervenants en soins palliatifs, des services de soutien à domicile des CLSC, du personnel des entreprises funéraires et des membres des Églises pour réfléchir à la manière dont nous pourrions mieux baliser un parcours rituel. À quand le temps où nous serons en mesure d'assurer un continuum de services et de sens en matière de ritualité pour ceux qui se préparent à mourir et ceux qui les soutiennent?

\section{Bibliographie}

AUGÉ, M. (1995). La mort et moi et nous, Paris, Textuel.

BACQUÉ, M.-F. (1997). Mourir aujourd'hui. Les nouveaux rites funéraires, Paris, Odile Jacob.

BASTIDE, R. (1975). Le sacré sauvage et d'autres essais, Paris, Payot.

BOUCHARD, G. (1992). "Sur les rituels de la mort au Saguenay (1880-1920) », Folklore canadien, vol. 14, $\mathrm{n}^{\circ} 1$, p. 123-139.

DOYON, M. (1954). «Rites de la mort dans la Beauce», Journal of American Folklore, vol. $67, \mathrm{n}^{\circ} 264$.

DURAND, G. (1992). Les structures anthropologiques de l'imaginaire, $11^{\mathrm{e}}$ édition, Paris, Dunod.

JEFFREY, D. (1998). Jouissance du sacré. Religion et postmodernité. Paris, Armand Colin.

JEFFREY, D. (2003). Éloge des rituels, Québec, Presses de l'Université Laval.

LEMIEUX, R. (1995). "La ritualité insoupçonnée », Liturgie, Foi et Culture, vol. 29, $\mathrm{n}^{\circ} 142$, p. 31-40.

LEBRETON, D. (2000). «Tatouages, piercings : rite personnel de passage?", Cultures en mouvement, $\mathrm{n}^{\circ} 29$, "Quels rites aujourd'hui ? », Antibes, juillet-août.

MÉNARD, G. (1999). Petit traité de la vraie religion à l'usage de ceux et celles qui souhaitent comprendre un peu mieux le vingt et unième siècle, Montréal, Liber.

GENNEP, A.V. (1981). Les rites de passage. Paris, Picard.

GOGUEL d'ALLONDAS, T. (1994). Rites de passage : d'ailleurs, ici pour ailleurs, RamonvilleSaint-Agne, Érès.

ST-ONGE, S. (2001). L'industrie de la mort, Québec, Nota bene.

THOMAS, L.V.(1985). Rites de mort... pour la paix des vivants, Paris, Fayard.

TURNER, V. (1990). Le phénomène rituel: structure et contre-structure, traduit de l'anglais par Gérard Guillet, Paris, Presses universitaires de France.

\section{Notes}

1. Pour la première collecte de données, nous avons fixé notre échantillonnage à une dizaine de familles. Il s'agit de décès par mort subite ou accidentelle. Au préalable, il avait été mentionné lors de nos discussions que ces familles ont tendance à diminuer les choix liés à la disposition du corps et aux différents gestes rituels à poser étant donné l'aspect non prévisible et le choc de la nouvelle du décès.

2. Nous tenons ici à souligner le travail accompli par une étudiante à la maîtrise en théologie pratique, Mme Audrey Houde, dont le mémoire de maîtrise, qui sera vraisemblablement déposé en juin 2004, est très lié à cette pratique. Mme Houde, sous la supervision de Mme Nicole Bouchard, a mené les différentes interventions auprès des familles. 